\title{
LE PHYLUM DES ACANTHOCEPHALA
}

Troisième note

La Classe des PALAEACANTHOCEPHALA (Meyer 1931) (suite)

Par Yves J. Golvan

\section{Diagnose de la famille des PSEUdACANTHOCEPHalide \\ Petrotschenko 1956}

Cette famille se compose du seul genre Pseudacanthocephalus Petrotschenko 1956. Nous ne possédons aucun renseignement, ni sur l'un, ni sur l'autre, la diagnose devant paraitre dans le second tome de la Monographie des Acanthocéphales de Petrotschenko.

\section{Diagnose de la famille des PLAGiORHYNCHIDA nov. fam.}

Palaeacanthocephala; parasites d'Oiseaux ou, plus rarement, de Reptiles, dont les hôtes d'attente sont des Vertébrés aquatiques ou terrestres et les hôtes intermédiaires des Insectes ou des Crustacés. Corps de taille petite à moyenne. Tronc inerme, fusiforme ou cylindroïde. Sous-cuticule contenant de nombreux fragments de noyaux géants. Canaux principaux du système lacunaire latéraux unis par des anastomoses transversales ou en réseau à mailles grossièrement polyédriques. Proboscis assez court, armé de files longitudinales de crochets, dont la taille décroît progressivement de l'apex à la base du rostre. Réceptacle à double paroi inséré à la base du proboscis. Place du ganglion cérébroïde variable, mais jamais dans la partie antérieure du réceptacle. Lemnisci de longueur variable. Embryophores de morphologie variable selon que le cycle est terrestre ou aquatique.

GenRe TYPE : Plagiorhynchus Lühe 1911.

Cette famille doit être scindée en trois sous-familles :

Plagiorhynchinæ Meyer 1931, Sphaerechinorhynchinæ nov. sub. fam., Porrorchinæ Golvan 1956. 


\section{Diagnose de la sous-Famille des Plagiorhynchinæ Meyer 1931}

Plagiorhynchida ; parasites d'Oiseaux aquatiques ou terrestres. Corps de taille petite à moyenne. Tronc inerme, fusiforme. Proboscis toujours assez long, cylindroïde, à grand axe fortement incliné ventralement sur le grand axe du tronc. Crochets disposés selon des files longitudinales et dont la taille diminue très progressivement de l'apex à la base du rostre. Les crochets supérieurs possèdent des racines bien développées, de forme généralement simple, et ont pratiquement tous la même taille. Les crochets inférieurs sont des épines à racines rudimentaires et leur nombre est toujours nettement inférieur à celui des crochets vrais. Réceptacle inséré à la limite du proboscis et du cou. Ganglion cérébroïde toujours situé à la partie moyenne du réceptacle. Lemnisci généralement beaucoup plus longs que le réceptacle. Organes mâles occupant plus de la moitié postérieure du tronc. Testicules sphéroïdaux ou ellipsoïdaux. Trois ou quatre glandes cémentaires parfois dédoublées. Orifices génitaux terminaux dans les deux sexes. Morphologie des embryophores variable selon que le cycle est aquatique ou terrestre.

\section{Genre type : Plagiorhynchus Lühe 1911.}

Cette sous-famille comprend, en outre, le genre Prosthorhynchus Kostylew 1915.

\section{Diagnose DU GEnRe Plagiorhynchus Lühe 1911}

Plagiorhynchinæ; parasites d'Oiseaux aquatiques ou subaquatiques se nourrissant d'Insectes ou de Crustacés. Corps de taille petite à moyenne. Tronc fusiforme, large chez les jeunes, plus allongé chez les adultes. Sous-cuticule contenant des noyaux de forme irrégulière et non sphéroïdaux comme ceux des Prosthorhynchus. Proboscis allongé, presque régulièrement cylindrique, armé de nombreux crochets, dont seuls les supérieurs possèdent des racines bien développées, alors que les basaux sont des épines à racines rudimentaires. Réceptacle inséré à la limite du cou et du proboscis. Ganglion placé au tiers moyen de la longueur du réceptacle. Lemnisci plus longs que le réceptacle. Organes mâles occupant les trois cinquièmes postérieurs de la longueur du tronc. Testicules sphéroïdaux, placés l'un derrière l'autre, parfois obliquement et en contact. Quatre glandes cémentaires longues et tubuleuses. Orifice génital terminal dans les deux sexes. Embryophores ellipsoïdaux à coque mince avec grandes hernies polaires de la membrane moyenne (ces hernies n'apparaissent que dans les embryophores parfaitement mûrs).

EsPÈcE TYPE : Plagiorhynchus crassicollis (Villot 1875) (= Echinorhynchus inflatus Creplin $1829=E$. crassicollis Villot $1875=$ 
E. lanceolatus von Linstow $1876=$ Plagiorhynchus lanceolatus (von Linstow 1876) Lühe $1911=$ Plagiorhynchus crassicollis (Villot 1875) Travassos 1926), parasite de Charadriiformes européens.

$2^{\circ}$ Plagiorhynchus crassicollis odhneri Lundström 1942 (= Plagiorhynchus odhneri Lundström 1942), parasite de Charadriiformes de Scandinavie.
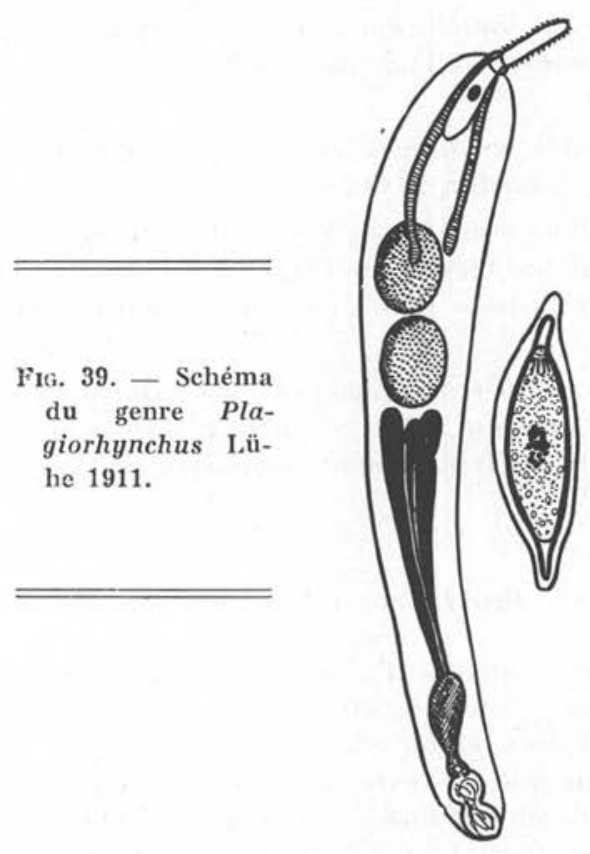

$3^{\circ}$ Plagiorhynchus charadriicola (Dollfus 1953) Golvan 1956 (= Prosthorhynchus charadriicola Dollfus 1953), parasite de Charadriiformes d'Afrique du Nord.

$4^{\circ}$ Plagiorhynchus rectus (Linton 1892) Van Cleave 1918 [ = Echinorhynchus rectus Linton 1892 = Prosthorhynchus rectus (Linton 1892) Travassos 1926], parasite d'une Mouette du Mexique.

$5^{\circ}$ Plagiorhynchus paulus Van Cleave et Williams 1950, parasite d'Oiseaux d'Alaska.

$6^{\circ}$ Plagiorhynchus linearis (Westrumb 1821) Golvan $1956[=$ Echinorhynchus sternæ Rudolphi 1819 nom. nud. = Echinorhynchus linearis Westrumb 1821 = Prosthorhynchus (?) linearis Meyer 1933], parasite d'Oiseaux aquatiques d'Europe.

$7^{\circ}$ Plagiorhynchus spiralis (Rudolphi 1809) Golvan $1956[=$ Echino- 
rhynchus spiralis Rudolphi 1802 = Prosthorhynchus spiralis (Rudolphi 1809) Meyer 1933], parasite d'Ardéiformes d'Europe.

$8^{\circ}$ Plagiorhynchus totani (Porta 1910) Golvan 1956 [= Echinorhynchus totani Porta 1910 = Prosthorhynchus totani (Porta 1910) Meyer 1933 ], parasite d'Oiseaux aquatiques d'Europe.

$9^{\circ}$ Plagiorhynchus charadrii Yamaguti 1939, parasite de Charadriiformes d'Extrême-Orient et d'Océanie.

$10^{\circ}$ Plagiorhynchus limnobaeni (Tubangui 1933) Golvan 1956 (= Prosthorhynchus limnobaeni Tubangui 1933), parasite d'Oiseaux des Philippines.

11' Plagiorhynchus menuræ (Johnston 1912) Golvan 1956 (= Prosthorhynchus menuræ Johnston 1912), parasite d'Oiseau-lyre d'Australie.

$12^{\circ}$ Plagiorhynchus reticulatus (Westrumb 1821) Golvan 1956 [ = Echinorhynchus reticulatus Westrumb 1821 = Prosthorhynchus reticulatus (Westrumb 1821) Travassos 1926], parasites d'Oiseaux aquatiques d'Amérique du Sud.

13 $^{\circ}$ Plagiorhynchus urichi (Cameron 1936) Golvan 1956 (= Prosthorhynchus urichi Cameron 1936), parasite trouvé accidentellement chez un Raton-laveur de la Trinité dont l'appartenance au genre est très incertaine.

\section{Diagnose du genre Prosthorhynchus Kostylew 1915}

Plagiorhynchinæ; parasites d'Oiseaux terrestres, en règle, insectivores stricts, dont les hôtes intermédiaires sont certainement des Insectes ou des Crustacés terrestres, et que l'on trouve souvent, accidentellement égarés, chez des Mammifères insectivores. Corps de taille moyenne. Tronc inerme, cylindroïde ou fusiforme. Proboscis cylindrique armé de files longitudinales de crochets dont la taille décroît progressivement de l'apex à la base du rostre. Les quelques crochets placés à la base des files sont des épines à racines rudimentaires, les autres sont des crochets vrais à racines bien développées. Réceptacle inséré à la limite entre le proboscis et le cou. Ganglion cérébroïde placé au tiers moyen du réceptacle. Lemnisci filiformes toujours bien plus longs que le réceptacle. Organes mâles occupant les trois quarts postérieurs de la longueur du tronc. Testicules sphéroïdaux, placés l'un derrière l'autre et souvent faiblement en contact. Trois glandes cémentaires longues et tubuleuses. Vulve termino-ventrale s'ouvrant au sommet d'une papille. Embryophores ovoïdes, à coque externe épaisse et souvent sculptée, jamais de hernies polaires de la membrane moyenne.

EsPÈce TYPE : Prosthorhynchus cylindraceus (Goeze 1782) Golvan 1956 (= Echinorhynchus cylindraceus Goeze $1782=E$. pici Gmelin $1791=E$. transversus Rudolphi $1819=E$. muscicapa Rudolphi 

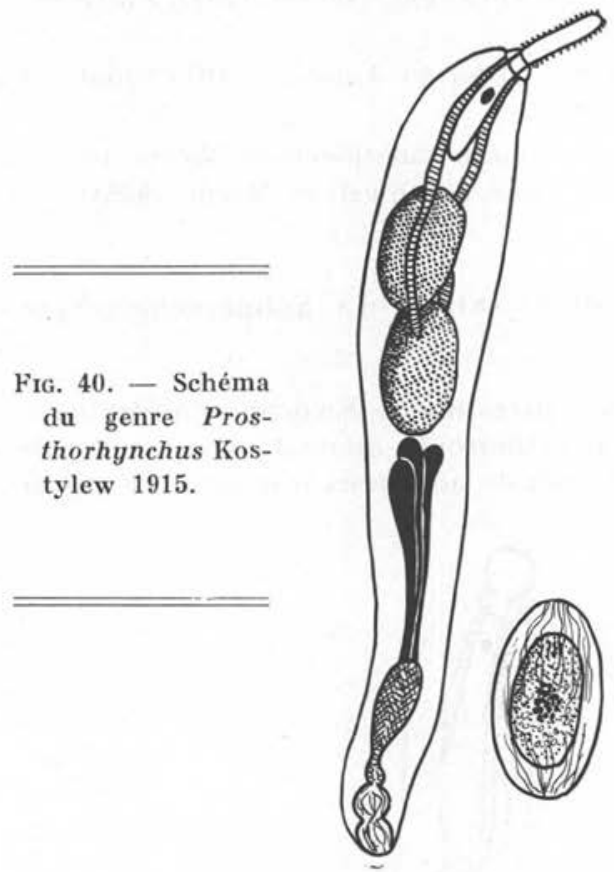

$1819=$ E. dimorphocephalus Westrumb $1821=E$. obliquus Dujardin $1845=E$. parvus Fuhrmann $1900=E$. pigmentatus de Marval $1902=E$. rostratus de Marval 1902 = Prosthorhynchus rosai $($ Porta 1910) $=$ Echinorhynchus brumpti Blanc et Cauchemez 1911 $=$ Prostorhynchus upupæ Lopez-Neyra $1946=P$. genitopapillatus Lundström 1942), parasite de Passériformes d'Europe, que l'on trouve très fréquemment égaré chez des Mammifères insectivores (Hérissons, Taupes, Musaraignes, etc...).

$2^{\circ}$ Prosthorhynchus rossicus Kostylew 1915, parasite de Vanneau de Russie (la détermination exacte de l'hôte n'est pas certaine).

$3^{\circ}$ Prosthorhynchus scolopacidis Kostylew 1915, parasite de la Bécasse d'Europe.

$4^{\circ}$ Prosthorhynchus formosus (Van Cleave 1918) Travassos 1926, parasite de Passériformes d'Amérique du Nord.

$5^{\circ}$ Prosthorhynchus longirostris Travassos 1926, parasite de Passériformes d'Amérique du Sud.

$6^{\circ}$ Prosthorhynchus angrense Travassos 1936, parasite de Passériformes d'Amérique du Sud. 
$7^{\circ}$ Prosthorhynchus rheæ (De Marval 1902) Travassos 1926, parasite de Rhea americana.

$8^{\circ}$ Prosthorhynchus pittarum Tubangui 1935, dont l'appartenance au genre est incertaine.

$9^{\circ}$ Prosthorhynchus bazæ (Southwell et Macfie 1925) Travassos 1926 (= Echinorhynchus bazæ Southwell et Macfie 1925).

\section{Diagnose DE LA SOUS-FAMILLE DES Sphaerechinorhynchinæ}

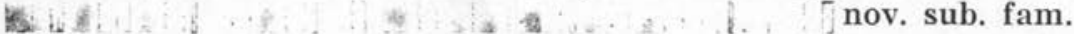

Plagiorhynchidæ; parasites de Serpents d'Australie. Corps de taille assez grande. Tronc cylindroïde, dilaté dans sa partie antérieure, inerme. Sous-cuticule contenant de nombreux fragments de noyaux géants placés

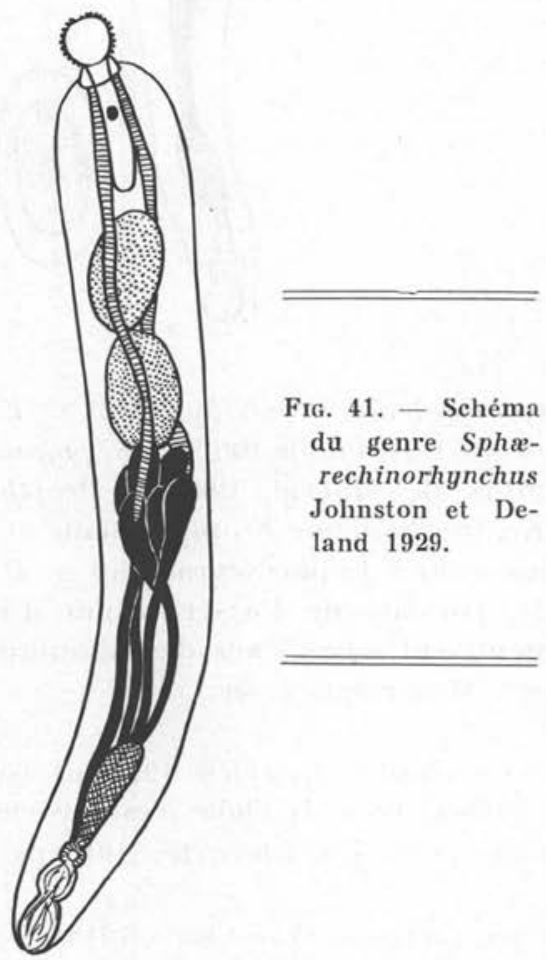

au milieu des lacunes. Canaux principaux du système lacunaire latéraux unis par des anastomoses formant un réseau à larges mailles grossièrement polyédriques. Proboscis court, sphéroïdal, armé de files longitudinales de crochets dont la taille diminue progressivement de haut en bas 
des files. Réceptacle à double paroi inséré à la base du rostre. Ganglion cérébroïde placé au tiers moyen de la hauteur du réceptacle. Lemnisci très longs, atteignant le testicule postérieur. Organes mâles occupant les quatre cinquièmes postérieurs de la longueur du tronc. Testicules ovoïdes, placés l'un derrière l'autre et en contact. Six glandes cémentaires très longues. Vulve s'ouvrant entre deux lobes terminaux. Embryophores ellipsoïdaux à coque mince avec hernies polaires de la membrane moyenne.

GENRE TYPE : Sphaerechinorhynchus Johnston et Deland 1929.

\section{DIAGNOSE DU GENRE Sphaerechinorhynchus}

Johnston et Deland 1929

Elle se confond actuellement avec celle de la sous-famille.

EsPÈCE TYPE : Sphaerechinorhynchus rotundocapitatus (Johnston 1912) Johnston et Deland 1929 (= Echinorhynchus rotundocapitatus Johnston 1912), provenant d'Australie.

\section{Diagnose de la sous-Famille des Porrorchinæ Golvan 1956}

Plagiorhynchidæ; parasites d'Oiseaux. Corps de taille moyenne à assez grande. Tronc inerme, avec souvent un renflement net dans sa partie antérieure. Proboscis court, subglobuleux ou conique, armé de files longitudinales de crochets dont la taille diminue progressivement de l'apex à la base du rostre. Réceptacle inséré à la base du proboscis. Ganglion cérébroïde placé dans le tiers moyen ou le tiers inférieur du réceptacle. Lemnisci souvent très longs, parfois anormalement nombreux. Organes mâles occupant plus de la moitié de la longueur du tronc. Quatre glandes cémentaires. Embryophores de forme variable selon que le cycle est aquatique ou terrestre.

Genre Type : Porrorchis Fukui 1929.

Cette sous-famille comprend, en outre, les genres :

Pseudoporrorchis Joyeux et Baer 1935,

Oligoterorhynchus Monticelli 1914,

Lueheia Travassos 1919,

Pseudogordiorhynchus Golvan 1957. 


\section{Diagnose du genre Porrorchis Fukui 1929}

Porrorchinæ; parasites d'Oiseaux d'Extrême-Orient, dont les hôtes d'attente sont des Batraciens. Corps de taille assez grande. Tronc inerme, allongé, cylindroïde. Sous-cuticule contenant des fragments de noyaux géants petits et compacts, rares dans la partie antérieure du tronc. Canaux principaux du système lacunaire latéraux unis par des anastomoses formant un réseau à mailles grossièrement polyédriques. Proboscis ovoïde armé de files longitudinales de crochets, les crochets supérieurs sont des crochets vrais à racines bien développées, les crochets inférieurs sont des épines à racines rudimentaires, qui parfois cependant possèdent des apophyses de forme complexe, comme dans le genre Centrorhynchus. Cou très court. Réceptacle inséré à la limite du proboscis et du cou. Ganglion cérébroïde placé à la partie moyenne du réceptacle. Lemnisci très longs, presque filiformes. Organes mâles occupant la moitié postérieure de la longueur du tronc. Testicules ovoïdes, placés l'un derrière l'autre, faiblement en contact ou un peu séparés. Quatre longues glandes cémentaires tubulaires. Embryophores ovoides à coque épaisse sans hernies polaires de la membrane moyenne.

Espèce TYPE : Porrorchis elongatus Fukui 1929, de Héron du Japon.

$2^{\circ}$ Porrorchis ogatai Fukui et Morishita 1936, parasite de Turdidæ du Japon.

$3^{\circ}$ Porrorchis oti Yamaguti 1939, parasite de Rapaces nocturnes du Japon.

\section{Diagnose du genre Pseudoporrochis Joyeux et Baer 1935}

Porrorchinæ; parasites de Cuculliformes de l'Ancien Monde dont les hôtes d'attente sont des Batraciens anoures ou des Reptiles et les hôtes intermédiaires probablement des Insectes. On peut trouver ces Parasites égarés chez des Mammifères. Corps de taille assez grande. Tronc cylindroïde, inerme, un peu dilaté dans sa partie antérieure, dans les deux sexes et dans sa partie terminale chez les femelles sexuellement mûres. Proboscis sphéroïdal armé de files longitudinales de crochets dont la taille diminue progressivement de haut en bas des files, les crochets basaux étant des épines à racines petites, présentant parfois des apophyses rendant leurs formes complexes. Réceptacle inséré à la limite du cou et du proboscis. Ganglion cérébroïde placé à la partie moyenne du réceptacle. Lemnisci digitiformes nettement plus longs que le réceptacle. Organes mâles occupant les cinq sixièmes de la longueur du tronc. Testicules ovoïdes placés l'un derrière l'autre et généralement un peu séparés. 


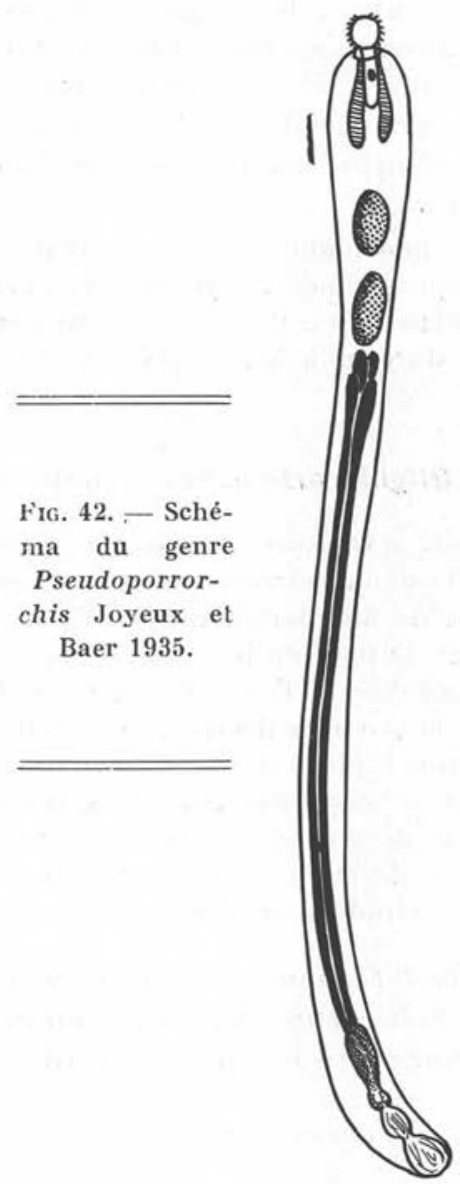

Quatre glandes cémentaires très longues et tubuleuses. Vulve terminoventrale avec appendice sus-vulvaire. Embryophores ovoïdes à coque épaisse et sculptée, dépourvus de hernies polaires de la membrane moyenne.

EsPèce TYPE : Pseudoporrorchis houdemeri Joyeux et Baer 1935, provenant d'Indochine.

$2^{\circ}$ Pseudoporrorchis rotundatus (Von Linstow 1897) Joyeux et Baer 1935, provenant de Madagascar.

$3^{\circ}$ Pseudoporrorchis centropi (Porta 1910) Joyeux et Baer 1935, provenant d'Afrique Occidentale.

$4^{\circ}$ Pseudoporrorchis hylæ (Johnston 1912) Edmonds 1956 [= Echino- 
rhynchus hylæ Johnston 1912 = Pseudoporrorchis bulbocaudatus (Southwell et Macfie 1925) Joyeux et Baer $1925=$ Gordiorhynchus hylæ (Johnston 1912) Johnston et Edmonds $1948=$ Pseudoporrorchis centropusi (Tubangui 1933)], provenant d'Australie et des Philippines.

$5^{\circ}$ Pseudoporrorchis teliger Van Cleave 1949, trouvé chez une Mangouste et un Chat de Java.

$6^{\circ}$ Pseudoporrorchis hydromuris Edmonds 1956, parasite d'un Rat d'eau d'Australie. L'appartenance au genre est incertaine, car il s'agit ici d'adultes sexuellement mûrs, parasites de Mammifères et non d'Oiseaux et les mâles ont six et non quatre glandes cémentaires.

\section{DIAGNOSE DU GENRE Oligoterorhynchus Monticelli 1914}

Porrorchinæ; parasite d'Oiseaux. Corps de taille moyenne. Tronc allongé, aplati, présentant un rétrécissement au tiers moyen, inerme. Proboscis ovoïde, armé de files longitudinales de crochets dont la taille décroît progressivement de haut en bas, les crochets inférieurs étant des épines à racines rudimentaires. Cou très long, cylindroïde, inséré sur une portion effilée de la partie antérieure du tronc. Réceptacle assez court. Ganglion cérébroïde placé à la partie moyenne du réceptacle. Lemnisci plus longs que le réceptacle, filiformes. Organes mâles occupant les deux tiers postérieurs de la longueur du tronc. Testicules ellipsoïdaux, placés obliquement l'un derrière l'autre et séparés. Quatre glandes cémentaires longues et cylindriques. Embryophores inconnus.

EsPÈcE TYPE: Oligoterorhynchus campylurus (Nitzsch 1857) Monticelli 1914 (=Echinorhynchus campylurus Nitzsch 1857), parasite d'un Stercoraire de l'Europe du Nord.

$2^{\circ}$ Oligoterorhynchus malayensis Tubangui 1935, parasite d'un Oiseau des Philippines.

\section{Diagnose DU genre Luehela Travassos 1919}

Porrorchinæ; parasites d'Oiseaux, dont les hôtes d'attente sont des Batraciens ou des Reptiles, de taille moyenne. Tronc allongé fusiforme, inerme. Proboscis court, subglobuleux, armé de files longitudinales de crochets dont la taille décroît progressivement de haut en bas. Cou bien marqué. Réceptacle assez long. Place du ganglion cérébroïde non précisée. Lemnisci plus longs que le réceptacle pouvant être en nombre anormalement élevé (six au lieu de quatre). Organes mâles occupant les trois quarts postérieurs de la longueur du tronc. Testicules ellipsoïdaux, placés l'un derrière l'autre et en contact. Quatre glandes cémentaires piriformes, allongées. Embryophores ellipsoïdaux à hernies polaires. 


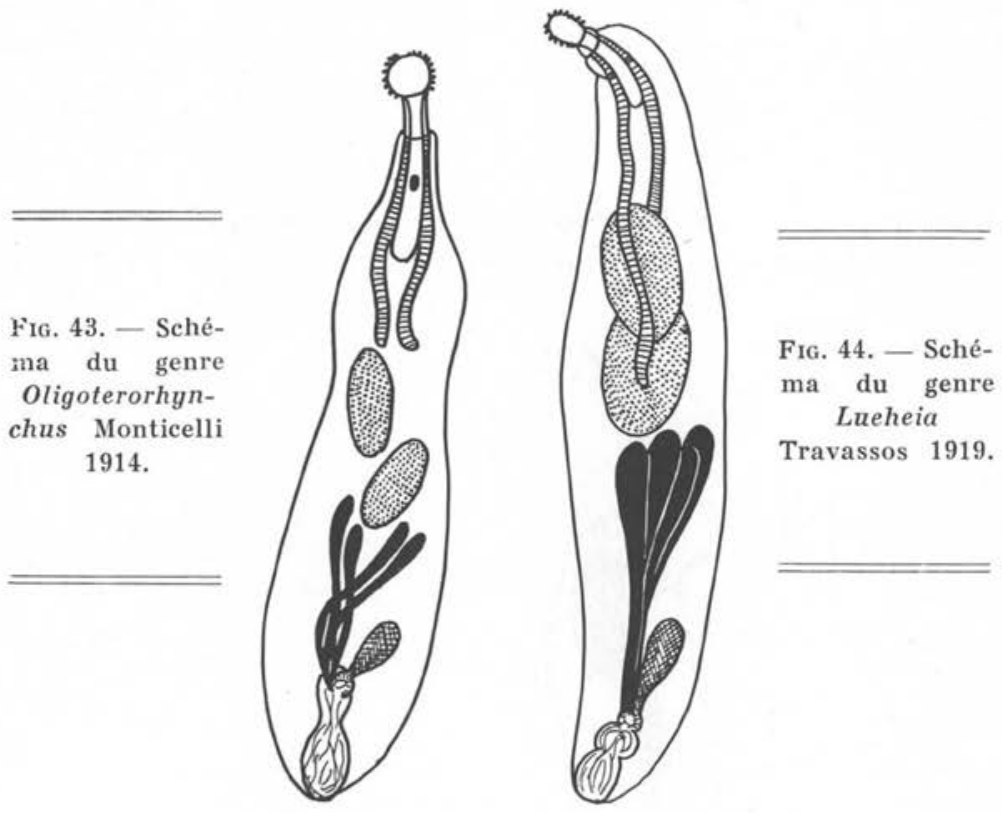

EsPÈCE TYPE : Lueheia luehei Travassos 1919, parasite d'Oiseaux d:Amérique du Sud.

$2^{\circ}$ Lueheia inscripta (Westrumb 1821) [ = Echinorhynchus inscriptus Westrumb $1821=$ Lueheia inscripta (Westrumb 1821) Travassos 1926], parasite de Turdidæe brésiliens.

\section{Diagnose du Genre Pseudogordiorhynchus Golvan 1957}

Porrorchinæ; parasites d'Alcedinidæ. Corps de taille moyenne. Tronc inerme, cylindroïde, un peu dilaté dans sa partie antérieure. Canaux principaux du système lacunaire latéraux unis par des anastomoses formant un réseau à larges mailles grossièrement polyédriques. Proboscis tronc-conique armé de files longitudinales de crochets dont la taille diminue progressivement de l'apex à la base du rostre. Les crochets supérieurs ont des racines bien développées. Les crochets inférieurs sont des épines à racines rudimentaires, dont le nombre est sensiblement égal à celui des crochets vrais. Réceptacle à double paroi inséré à la limite du proboscis et du cou. Ganglion cérébroïde situé sensiblement à la partie moyenne du réceptacle. Lemnisci digitiformes nettement plus longs que le réceptacle. Organes mâles occupant les quatre cinquièmes

Ann. de Parasitologie, T. XXXV, $\mathrm{N}^{\circ} 3 .-1960$. 
postérieurs de la longueur du tronc. Testicules ovoïdes situés l'un derrière l'autre, plus ou moins en contact. Trois glandes cémentaires longues et tubulaires. Orifices génitaux terminaux dans les deux sexes. Embryophores ovoïdes à coque assez épaisse avec ébauches de hernies polaires de la membrane moyenne.

ESPÈCE TYPE : Pseudogordiorhynchus antonmeyeri Golvan 1957. parasite d'Alcédinidés africains.

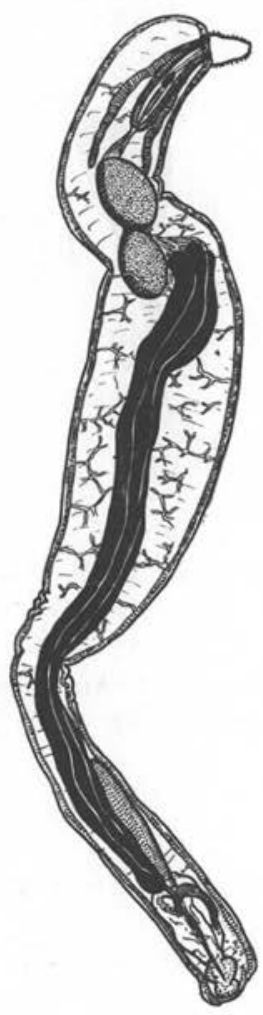

FIG. 45. - Schéma du genre Pseudogordiorhynchus Golvan 1957.

Diagnose de la famille des CENTRORHYNChide nov. fam.

Palaeacanthocephala; parasites d'Oiseaux terrestres, dont les hôtes intermédiaires sont des Insectes, et les hôtes d'attente des Batraciens, des Reptiles, voire des Mammifères insectivores. Corps de taille moyenne à assez grande. Tronc inerme, fusiforme ou cylindroïde. Sous-cuticule contenant de nombreux fragments de noyaux géants. Canaux principaux du système lacunaire latéraux unis, soit par des anastomoses transver- 
sales, soit par un réseau à mailles grossièrement polyédriques. Proboscis tronc-conique toujours formé de deux portions superposées séparées par une striction. Crochets disposés selon des files ventrales dont la taille décroît de l'apex à la base du rostre. Les crochets supérieurs sont des crochets vrais à racines bien développées. Les crochets inférieurs sont des épines à racines rudimentaires. Il existe parfois des crochets de transition, à racines courtes et compliquées d'apophyses de formes diverses. Réceptacle à double paroi inséré à la partie moyenne du proboscis, au niveau de sa partie rétrécie. Ce caractère est celui qui permet de différencier le plus facilement les Centrorhynchidæ de tous les autres Palaeacanthocephala. Ganglion cérébroïde placé à la partie moyenne du réceptacle. Lemnisci digitiformes toujours plus longs que le réceptacle. Organes mâles occupant toujours plus de la moitié postérieure de la longueur du tronc. Trois (quatre ?) glandes cémentaires longues tubuleuses ou en massue à grosse extrémité supérieure. Embryophores caractèristiques d'un cycle terrestre.

GENRE TYPE : Centrorhynchus Lühe 1911.

La famille comprend, en outre, le genre Sphaerirostris (Golvan 1956). Eu égard au nombre des espèces décrites, cette famille est la plus importante de tout le Phylum des Acanthocephala.

\section{Diagnose du genRe Centrorhynchus Lühe 1911}

\section{$(=$ Paradoxites Lindemann $1865=$ Echinorhynchus $\quad$ (Zoega)}

O.F. Müller $1780=$ Chentrosoma Monticelli 1905 p.p. $=$ Chentrosoma Porta 1909 p.p. = Gordiorhynchus Meyer 1931).

Centrorhynchidx; parasites de Rapaces diurnes ou nocturnes dont les hôtes intermédiaires sont des Insectes (Acridiens) et les hôtes d'attente des Batraciens, des Reptiles ou des Mammifères insectivores. Corps de taille moyenne à assez grande. Tronc cylindroïde souvent nettement dilaté dans sa partie antérieure, inerme. Canaux principaux du système lacunaire latéraux unis par des anastomoses transversales. Proboscis assez long formé de deux portions tronc-coniques superposées à bases inférieures. Crochets disposés en files longitudinales. Crochets supérieurs grands à racines bien développées dont la taille croît progressivement de haut en bas. Crochets inférieurs à racines rudimentaires qui sont en fait des épines. Il existe souvent, à la partie moyenne du proboscis, des crochets de forme intermédiaire à racines courtes et larges dont la forme est compliquée par la présence d'apophyses. Réceptacle à double paroi inséré à l'union des deux portions du proboscis. Ganglion cérébroïde placé à la partie moyenne du réceptacle. Lemnisci digitiformes toujours plus longs que le réceptacle. Organes mâles occupant au moins les quatre 


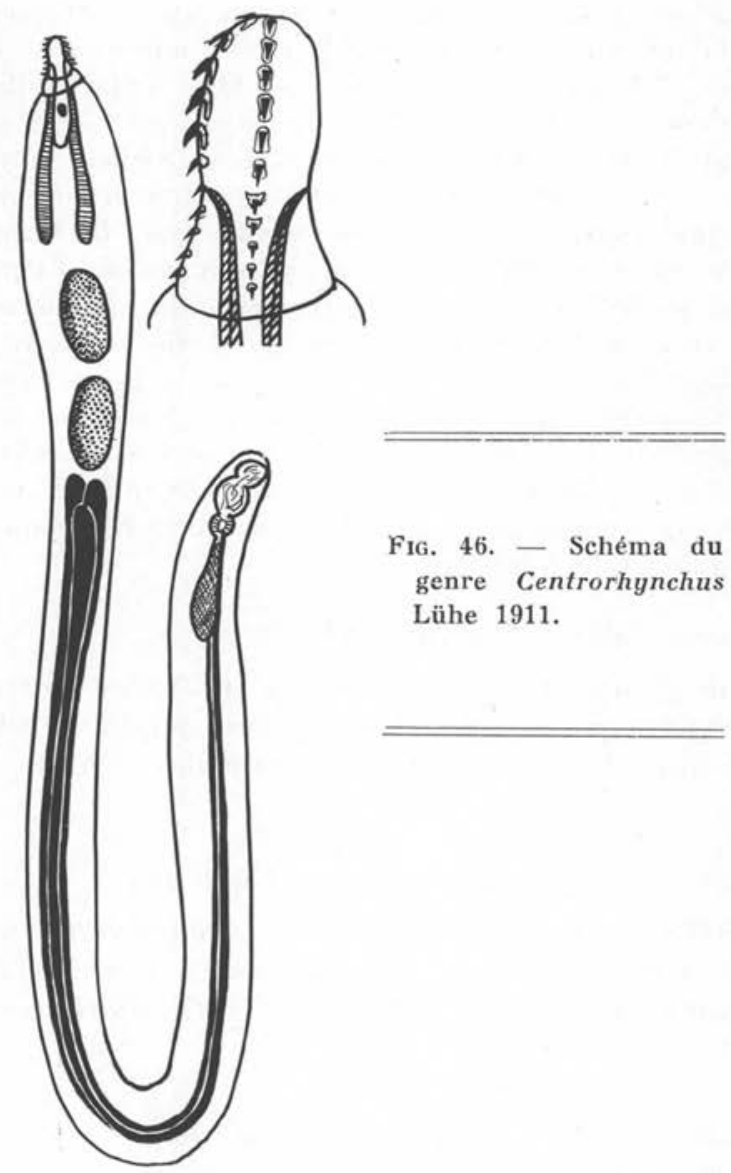

cinquièmes de la longueur du tronc. Testicules ovoïdes placés l'un derrière l'autre, séparés ou en contact. Trois (quatre ?) glandes cémentaires longues et tubuleuses souvent difficilement discernables les unes des autres. Orifice mâle terminal. Vulve ventro-terminale avec souvent un appendice sus-vulvaire. Embryophores ovoïdes à coque externe épaisse et sculptée, pas de hernies polaires de la membrane moyenne.

EsPÈCE TYPE : Centrorhynchus aluconis (O. F. Müller 1780) (= Echinorhynchus aluconis O. F. Müller $1780=$ E. mergi Bloch 1782 $=E$. tuba Rudolphi 1802 in Creplin $1848=E$. otidis Schrank 1788 -E. inequalis Rudolphi $1808=E$. polyacanthoides Creplin 1825 $=E$. appendiculatus Westrumb $1821=E$. soricis Rudolphi $1819=$ E. mirabilis Polonio $1859=E$. contortus Molin $1861=E$. croati- 
cus Stossich $1899=$ E. bacillaris Zeder 1803 p.p. = Chentrosoma aluconis Porta $1909=$ Centrorhynchus globocaudatus Zeder 1800 $=$ C. scanensis Lundström $1942=$ C. olssoni Lundström 1942), parasite de Rapaces diurnes et nocturnes d'Europe.

$2^{\circ}$ Centrorhynchus conspectus Van Cleave et Pratt 1940, parasite de Hibou d'Amérique du Nord.

$3^{\circ}$ Centrorhynchus elongatus Yamaguti 1935, parasite de Hiboux du Japon.

$4^{\circ}$ Centrorhynchus bubonis Yamaguti 1939, parasite de Hibou du Japon.

$5^{\circ}$ Centrorhynchus buteonis (Schrank 1788) $(=$ Echinorhynchus buteonis Schrank $1788=E$. caudatus Zeder $1803=E$. haliaeti Rudolphi 1819 $=E$. polyacanthoides Creplin $1825=E$. tenuicaudatus Marotel 1899), parasite de Rapaces d'Europe.

$6^{\circ}$ Centrorhynchus clitorideus (Meyer 1931) (= Gordiorhynchus clitorideus Meyer 1931), parasite de Hibou d'Europe Orientale.

$7^{\circ}$ Centrorhynchus leptorhynchus Meyer 1932 (= Echinorhynchus cinctus Rudolphi 1819 p.p.), qui n'est peut-être qu'un juvénile de l'espèce précédente.

$8^{\circ}$ Centrorhynchus ninni (Stossich 1891) (= Echinorhynchus ninni Stossich $1891=$ Echinorhynchus sp. Perroncito $1882=$ Chentrosoma ninni), parasite trouvé chez un Putois d'Italie et dont l'hôte définitif normal doit être un Rapace.

$9^{\circ}$ Centrorhynchus lesiniformis (Molin 1859) (= Echinorhynchus lesiniformis Molin 1859), connu par ces juvéniles trouvés chez des Batraciens et des Reptiles d'Europe.

$10^{\circ}$ Centrorhynchus narcissa Florescu 1942, parasite de Rapaces de Roumanie.

$11^{\circ}$ Centrorhynchus bancrofti (Johnston et Best 1943) (= Gordiorhynchus bancrofti Johnston et Best 1943), parasite de Rapaces d'Australie.

$12^{\circ}$ Centrorhynchus batrachus Das 1952, parasite trouvé à l'état de juvéniles chez des grenouilles des Indes.

$13^{\circ}$ Centrorhynchus spinosus (Kaiser 1893) (= Echinorhynchus spinosus Kaiser 1893), parasite de Rapaces d'Amérique du Nord et des Galapagos.

$14^{\circ}$ Centrorhynchus polymorphus Travassos 1926, parasite de Rapaces d'Amérique du Sud.

$15^{\circ}$ Centrorhynchus indicus Golvan 1956 (= Centrorhynchus falconis Das 1949), parasite de Rapaces des Indes.

$16^{\circ}$ Centrorhynchus brevicanthus Das 1949 , parasite de Rapaces des Indes. 
$17^{\circ}$ Centrorhynchus undulatus R.-Ph. Dollfus 1951, parasite de Rapaces du Maroc, peut-être synonyme d'Echinorhynchus undulatus (Nitzsch in Giebel 1857.

$18^{\circ}$ Centrorhynchus milvus Ward 1956, parasite du Milan noir d'Afrique.

$19^{\circ}$ Centrorhynchus asturinus (Johnston 1913) (= Gigantorhynchus asturinus Johnston 1913), parasite de Rapaces d'Australie.

$20^{\circ}$ Centrorhynchus insularis Tubangui 1933, parasite de Rapaces diurnes des Philippines.

$21^{\circ}$ Centrorhynchus itatsinis Fukui 1929, parasite trouvé chez un Mustellidæ du Japon.

$22^{\circ}$ Centrorhynchus magnus Fukui 1929 (= Centrorhynchus micror chis Fukui 1929), parasite de Rapaces et accidentellement de Héron du Japon.

$23^{\circ}$ Centrorhynchus falconis (Johnston et Best 1943) (= Gordiorhynchus falconis Johnston et Best 1943), parasite de Rapaces d'Australie.

$24^{\circ}$ Centrorhynchus microcephalus (Bravo Hollis 1947) (= Gordiorhynchus microcephalus Bravo Hollis 1947), parasite d'Oiseaux du Mexique.

$25^{\circ}$ Centrorhynchus freundi (Hartwich 1953) (= Gordiorhynchus freundi Hartwich 1953), parasite d'un Secrétaire africain.

$26^{\circ}$ Centrorhynchus californicus Millzner 1924, parasite trouvé chez une Rainette d'Amérique du Nord à l'état de juvénile.

$27^{\circ}$ Centrorhynchus crocidurus Das 1950 , parasite trouvé à l'état de juvénile chez une Musaraigne des Indes.

$28^{\circ}$ Centrorhynchus galliardi Golvan 1956, parasite de Rapaces d'Afrique du Nord.

$29^{\circ}$ Centrorhynchus albidus Meyer 1933, parasite d'un Falconidæ du Paraguay.

$30^{\circ}$ Centrorhynchus tumidulus (Rudolphi 1819) (= Echinorhynchus tumi dulus Rudolphi $1819=E$. caudatus Rudolphi $1819=E$. megacephalus Westrumb $1821=$ Chentrorhynchus tumidulus Neiva Cuhna et Travassos 1914), parasite de Rapaces du Brésil.

$31^{\circ}$ Centrorhynchus giganteus Travassos 1919, parasite de Rapaces du Brésil.

$32^{\circ}$ Centrorhynchus simplex Meyer 1933, parasite décrit d'après des juvéniles trouvés chez un Reptile du Brésil.

$33^{\circ}$ Centrorhynchus horridus (Von Linstow 1897) [= Echinorhynchus horridus Von Linstow 1897 = Prosthorhynchus horridus (Von Linstow 1897) Travassos 1926], parasite d'un Alcédinidé de l'Archipel Bismarck. La similitude des hôtes nous avait, à tort, fait croire que cette espèce pouvait être un Pseudogordiorhynchus. Johnston et Edmonds (1948) ont 
redécrit l'espèce et indiquent formellement (tout comme Meyer d'ailleurs) que le réceptacle s'insère à la partie moyenne du proboscis. Il s'agit donc bien d'un Centrorhynchus.

$34^{\circ}$ Centrorhynchus dipsadis (Von Linstow 1888), décrit d'après des juvéniles trouvés chez un Serpent du Cameroun.

$35^{\circ}$ Centrorhynchus mabuize (Von Linstow 1908), décrit d'après des juvéniles trouvés chez un Serpent de Java.

$36^{\circ}$ Centrorhynchus macrorchis Das 1949, parasite d'un Rapace des Indes.

$37^{\circ}$ Centrorhynchus brevicaudatus Das 1949, parasite trouvé chez un Poisson des Indes (Ophiocephalus sp.), qui est un hôte de réenkystement tout à fait accidentel pour un Centrorhynchus.

$38^{\circ}$ Centrorhynchus amphibus Das 1950, parasite décrit d'après des juvéniles enkystés chez des Reptiles et des Batraciens Anoures des Indes.

$39^{\circ}$ Centrorhynchus ptyasus Gupta 1950, parasite décrit d'après des juvéniles provenant de Serpents des Indes.

$40^{\circ}$ Centrorhynchus longicephalus Das 1950, décrit d'après des juvéniles trouvés chez un Serpent.

$41^{\circ}$ Centrorhynchus microcerviacanthus Das 1950, décrit d'après des juvéniles trouvés chez un Serpent des Indes.

$42^{\circ}$ Centrorhynchus gendrei (Golvan 1957) (= Gordiorhynchus gendrei Golvan 1957), parasite de Rapaces d'Afrique Occidentale.

$43^{\circ}$ Centrorhynchus madagascariensis (Golvan 1957) (= Gordiorhyn . chus madagascariensis Golvan 1957), parasite de Rapaces de Madagascar.

$44^{\circ}$ Centrorhynchus chabaudi Golvan 1958, parasite de Rapaces d'Afrique Occidentale.

$45^{\circ}$ Centrorhynchus petrotschenkoi Kuraschvili 1955, parasite de Rapaces ou d'Ardéiformes (hôte accidentel ?) d'U.R.S.S.

$46^{\circ}$ Centrorhynchus bazaleticus Kurashvili 1955, parasite d'Ardéiformes (?) d'U.R.S.S.

De nombreuses espèces ont été décrites d'après des Juvéniles. Pour notre part, nous nous sommes refusé à donner un nom à ces juvéniles dont l'étude anatomique ne peut être que trop succincte, et dont les noms viennent encore compliquer une systématique déjà difficile.

\section{DIAGNOSE DU GENRE Sphaerirostris (Golvan 1956)}

Centrorhynchidx; parasites de Passériformes (Corvidæ ou Turdidæ) mais jamais de Rapaces (du moins normalement). Corps de taille petite à moyenne. Tronc inerme, fusiforme, large. Canaux principaux du système lacunaire latéraux unis par un réseau d'anastomoses à mailles 
grossièrement polyédriques. Proboscis assez court, formé de deux portions superposées : une portion supérieure sphéroïdale et une portion inférieure tronc-conique, armé de crochets dont la taille croît de l'apex à la limite entre les deux parties du rostre. Ces crochets supérieurs ont une racine bien développée de forme parfois complexe. Au-dessous de cette limite les crochets sont des épines à racines rudimentaires, de forme parfois compliquée par l'existence d'apophyses. Réceptacle à

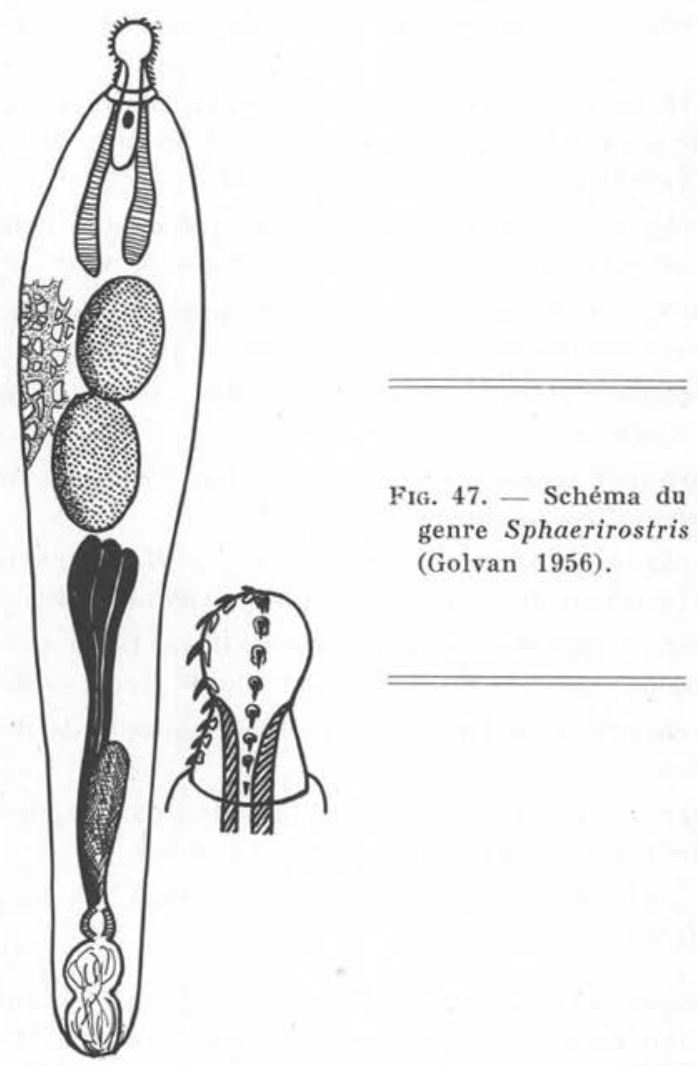

double paroi inséré à la limite des deux portions du rostre. Ganglion cérébroïde placé à peu près à la moitié de la hauteur du réceptacle. Lemnisci digitiformes, plus longs que le réceptacle. Organes mâles occupant les quatre cinquièmes postérieurs de la longueur du tronc. Testicules sphéroïdaux, placés l'un derrière l'autre et faiblement en contact. Trois (quatre) glandes cémentaires longues, en massue, à grosse extrémité supérieure. Orifices génitaux terminaux dans les deux sexes. Pas d'appendice sus-vulvaire. Embryophores ovoïdes à coque épaisse et souvent sculptée, sans hernies polaires de la membrane moyenne. 
EsPÈCE TYPE : Sphaerirostris picæ (Rudolphi 1819) Golvan 1958 (=Echinorhynchus picæ Rudolphi $1819=$ E. teres Westrumb 1921 $=E$. hepaticus Molin $1858=E$. lobianchii Monticelli $1887=$ Centrorhynchus picæ Dollfus 1953), parasite de Turdidæ d'Europe et d'Afrique du Nord.

$2^{\circ}$ Sphaerirostris opimus (Travassos 1919) (= Centrorhynchus opimus Travassos 1919), parasite d'un Tyrannidæ du Brésil.

$3^{\circ}$ Sphaerirostris areolatus (Rudolphi 1819) (= Echinorhynchus areolatus Rudolphi $1819=E$. sigmoides Westrumb $1821=E$. orioli Rudolphi 1819), parasite de Passériformes d'Europe.

$4^{\circ}$ Sphaerirostris lancea (Westrumb 1821) $[=$ Echinorhynchus vanelli (Goeze 1782) Gmelin 1790 nom. nud. $=E$. morinelli Rudolphi 1819 nom. nud. $=E$. oedicnemi Rudolphi 1819 nom. nud. $=E$. merualæ Gmelin 1791 p.p. $=$ E. cinctus Rudolphi 1819 p.p. = Centrorhynchus cinctus (Rudolphi 1819) Meyer $1933=$ C. embæ Kolodkowski et Kostylew 1916 $=$ C. scanensis Lundström 1942], parasite de Turdidæe et de Charadriiformes d'Europe.

$5^{\circ}$ Sphaerirostris turdi (Yamaguti 1939), parasite de Turdidæ du Japon (= Centrorkynchus turdi Yamaguti 1939).

$6^{\circ}$ Sphaerirostris lanceoides Petrotschenko 1949 (- Centrorhynchus lanceoides Petrotschenko 1949), parasite de Turdidæ d'U.R.S.S.

$7^{\circ}$ Sphaerirostris pinguis (Van Cleave 1918) (= Centrorhynchus leguminosus Solovieff $1912=$ C. bipartitus Solovieff $1912=$ Centrorhynchus corvi Fukui 1929 = C. skrjabini Petrotschenko 1949), parasite de Turdidæe d'Asie.

$8^{\circ}$ Sphaerirostris serpenticola (Von Linstow 1908) (= Echinorhynchus serpenticola Von Linstow 1908), décrit d'après des juvéniles enkystés chez un Viperide de Java.

$9^{\circ}$ Sphaerirostris physocoracis (Porta 1913), parasite d'un Oiseau de Nouvelle-Calédonie.

$10^{\circ}$ Sphaerirostris maryasis (Datta 1932) (= Centrorhynchus maryasis Datta 1932), parasite de Corvidæ des Indes.

$11^{\circ}$ Sphaerirostris reptans (Bhalerao 1931) (= Centrorhynchus reptans Bhalerao 1931), décrit sommairement d'après des juvéniles trouvés chez un Naja des Indes.

$12^{\circ}$ Sphaerirostris erraticus (Chandler 1925) (Centrorhynchus erraticus Chandler 1925), décrit d'après des adultes immatures égarés chez un Chat des Indes. 